\title{
Changes of alveolar bone dehiscence and fenestration after augmented corticotomy- assisted orthodontic treatment: a CBCT evaluation
}

\author{
Liangyan Sun ${ }^{1}$, Lingjun Yuan², Bo Wang ${ }^{3}$, Lina Zhang ${ }^{4}$, Guofang Shen ${ }^{3}$ and Bing Fang ${ }^{2 *}$ (D)
}

\begin{abstract}
Background: To evaluate the changes of alveolar dehiscence and fenestration after augmented corticotomyassisted orthodontic treatment on cone-beam computed tomography (CBCT) compared with traditional presurgical orthodontics, both quantitatively and qualitatively.

Methods: Two hundred and four anterior teeth from 17 skeletal class III malocclusions were divided into four groups. Groups G1 (upper teeth) and G3 (lower teeth), comprising 120 teeth, accepted traditional pre-surgical orthodontics; groups G2 (upper teeth) and G4(lower teeth), comprising 84 teeth, accepted augmented corticotomyassisted pre-surgical orthodontics. The changes of alveolar bone dehiscence and fenestration of each tooth in all groups were evaluated with the help of CBCT.

Results: Quantitative analysis for comparing both groups: For the upper teeth, $d_{1}-d_{0}$ was different between both groups while $f_{1}-f_{0}$ was not statistically different. For the lower teeth, $d_{1}-d_{0}$ was statistically different between both groups while $f_{1}-f_{0}$ was not statistically different. Qualitative analysis: For the teeth that had no dehiscence before treatment, G2 and G4 had a better transition than did G1 and G3. For those having dehiscence before treatment, G4 had a better transition than did G3. For teeth having no fenestration before treatment, there was no statistically significant difference in transition between the control and treatment groups. For those having fenestration before treatment, G4 had a better transition than did G3.

Conclusions: For skeletal class III patients, augmented corticotomy-assisted orthodontic treatment is a promising method of improving alveolar bone dehiscence and fenestration for lower anterior teeth, and it also has the potential to protect both lower and upper anterior teeth against dehiscence.
\end{abstract}

Keywords: Augmented corticotomy-assisted orthodontic treatment, Dehiscence, Fenestration, CBCT

\section{Background}

Naturally occurring alveolar bone dehiscence and fenestration are common findings in different types of malocclusions [1-5], especially in the anterior region of class III malocclusions [1, 5]. Dehiscence and fenestration may lead to gingival recession and additional bone loss during orthodontic treatment [6-8]. In addition, large

\footnotetext{
* Correspondence: fangbing@sjtu.edu.cn

${ }^{2}$ Department of Orthodontics, Ninth People's Hospital, College of

Stomatology, Shanghai Jiao Tong University School of Medicine, 500 Quxi Rd, 7th Floor, Shanghai 200000, China

Full list of author information is available at the end of the article
}

amount of labial inclination such as decompensation in class III malocclusions may pose a greater risk of periodontal complications, such as alveolar dehiscence and fenestration and gingival recession [9-12].

Since these disorders can complicate orthodontic treatment by causing gingival recession and additional bone loss, it is important that they be detected before treatment so that they can be treated or prevented. Timock et al. [13] found that the accuracy and reliability of buccal bone height and thickness measurements from CBCT are acceptable and appropriate. Leung et al. [14] found that the diagnostic value of $\mathrm{CBCT}$ for detecting 


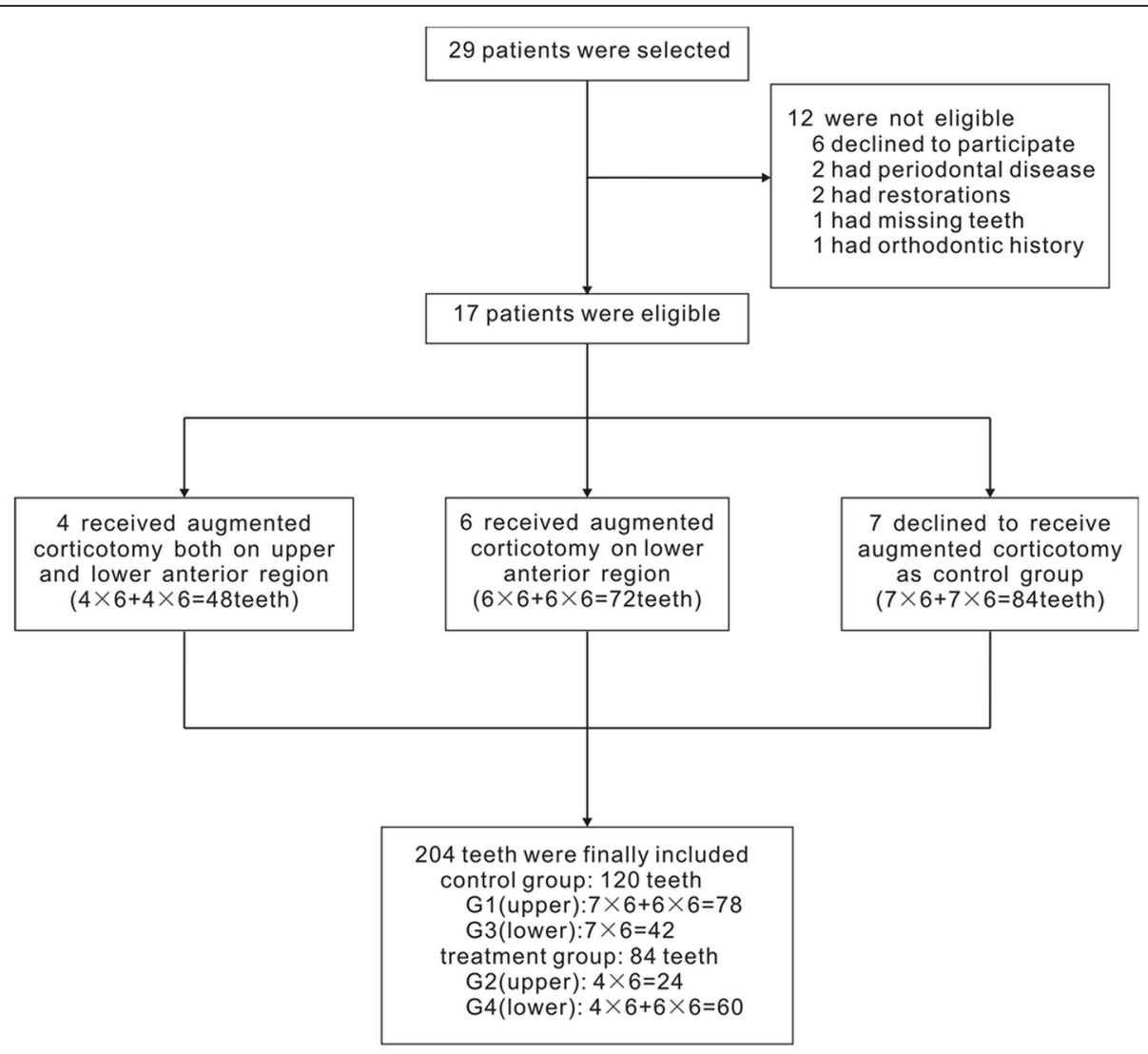

Fig. 1 Screening procedure of the selected samples

buccal defects was high for fenestrations. Sun et al. [15] proposed a CBCT method having a relatively high accuracy to diagnose alveolar dehiscence and fenestration. Nowadays, with CBCT widely used as an orthodontic pretreatment record, alveolar bone dehiscence and fenestration can be easily diagnosed.

Periodontally accelerated osteogenic orthodontics (PAOO) is a combination of bone activation, alveolar augmentation using particulate bone grafting material, and orthodontic treatment [16]. It is believed that PAOO can increase alveolar volume and cover vital root surfaces, which may result in repairing preexisting alveolar dehiscences and fenestrations [16-18]. Some recent studies have reported that alveolar bone thickness may increase after augmented corticotomy-assisted surgical orthodontics [19, 20]. Wang et al. [19] reported that the apical region had a larger amount of alveolar augmentation. Ma et al. [20] applied the "dumpling" technique to augmented corticotomy-assisted orthodontics, and the results showed that both vertical alveolar height and horizontal bone thickness increased in the labial aspect of the anterior mandibular area. Yu et al. [21] reported that alveolar fenestration and bony dehiscence could be successfully addressed after PAOO. However, Wang

Table 1 Demographic and pretreatment characteristics of the sample

\begin{tabular}{|c|c|c|c|c|c|c|}
\hline \multirow[t]{2}{*}{ Variable } & \multicolumn{2}{|l|}{ Maxillary } & \multirow[t]{2}{*}{$P$} & \multicolumn{2}{|l|}{ Mandibular } & \multirow[t]{2}{*}{$P$} \\
\hline & G1 & G2 & & $\mathrm{G} 3$ & G4 & \\
\hline Age, years & $20.4 \pm 2.1$ & $21.6 \pm 3.1$ & 0.424 & $20.3 \pm 2.0$ & $20.9 \pm 2.6$ & 0.676 \\
\hline ANB, degree & $-3.6 \pm 1.4$ & $-2.9 \pm 2.1$ & 0.508 & $-3.3 \pm 1.6$ & $-3.5 \pm 1.7$ & 0.821 \\
\hline Overjet, mm & $-1.9 \pm 2.0$ & $-0.8 \pm 0.7$ & 0.320 & $-1.6 \pm 1.1$ & $-1.7 \pm 2.2$ & 0.957 \\
\hline UI-SN, degree & $110.1 \pm 5.8$ & $115.9 \pm 7.9$ & 0.154 & $111.2 \pm 6.1$ & $111.8 \pm 7.4$ & 0.881 \\
\hline L1-MP, degree & $74.4 \pm 6.9$ & $76.6 \pm 11.1$ & 0.669 & $78.7 \pm 4.0$ & $72.3 \pm 9.2$ & 0.125 \\
\hline Crowding, mm & $2.0 \pm 0.7$ & $2.1 \pm 0.4$ & 0.849 & $2.0 \pm 0.8$ & $2.2 \pm 0.6$ & 0.740 \\
\hline Treatment time, years & $2.3 \pm 0.2$ & $1.6 \pm 0.2$ & 0.000 & $2.3 \pm 0.2$ & $2.0 \pm 0.3$ & 0.036 \\
\hline
\end{tabular}



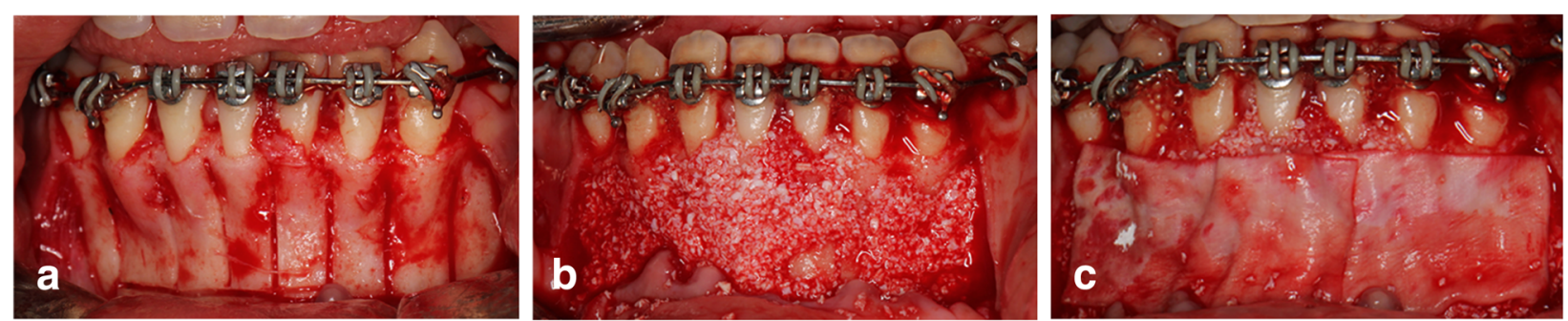

Fig. 2 The description of the bone activation and GBR. a Performance of selective alveolar decortication. b Place bovine inorganic bone over the anterior region. c Place collagen membrane over the bone graft material)

et al. [19] and Ma et al. [20] did not focus on the changes of alveolar dehiscence and fenestration, and no quantitative description was included in the $\mathrm{Yu}$ et al. study [21].

The aim of this study was to evaluate the CBCT-evident changes of alveolar dehiscence and fenestration after augmented corticotomy-assisted orthodontic treatment compared with traditional pre-surgical orthodontics, both quantitatively and qualitatively. The hypothesis was that the changes of alveolar bone dehiscence and fenestration are different after augmented corticotomy-assisted presurgical orthodontic treatment and conventional procedures.

\section{Methods}

\section{Subjects and samples}

Severe class III patients who were to undergo orthognathic surgery from March 2015 to September 2015 were selected from the Department of Oral \& Cranio-Maxillofacial Science, Ninth People's Hospital. Twenty-nine patients were selected according to the following inclusion criteria: (1) adult, (2) prepared to undergo orthognathic surgery (bilateral sagittal split ramus osteotomy, Le Fort I osteotomy, or both), (3) mild dental crowding $(0-3 \mathrm{~mm})$, and (4) clinical and $\mathrm{CBCT}$ examination indicating potential dehiscence and fenestration or thin alveolus and prominent root(s) in the

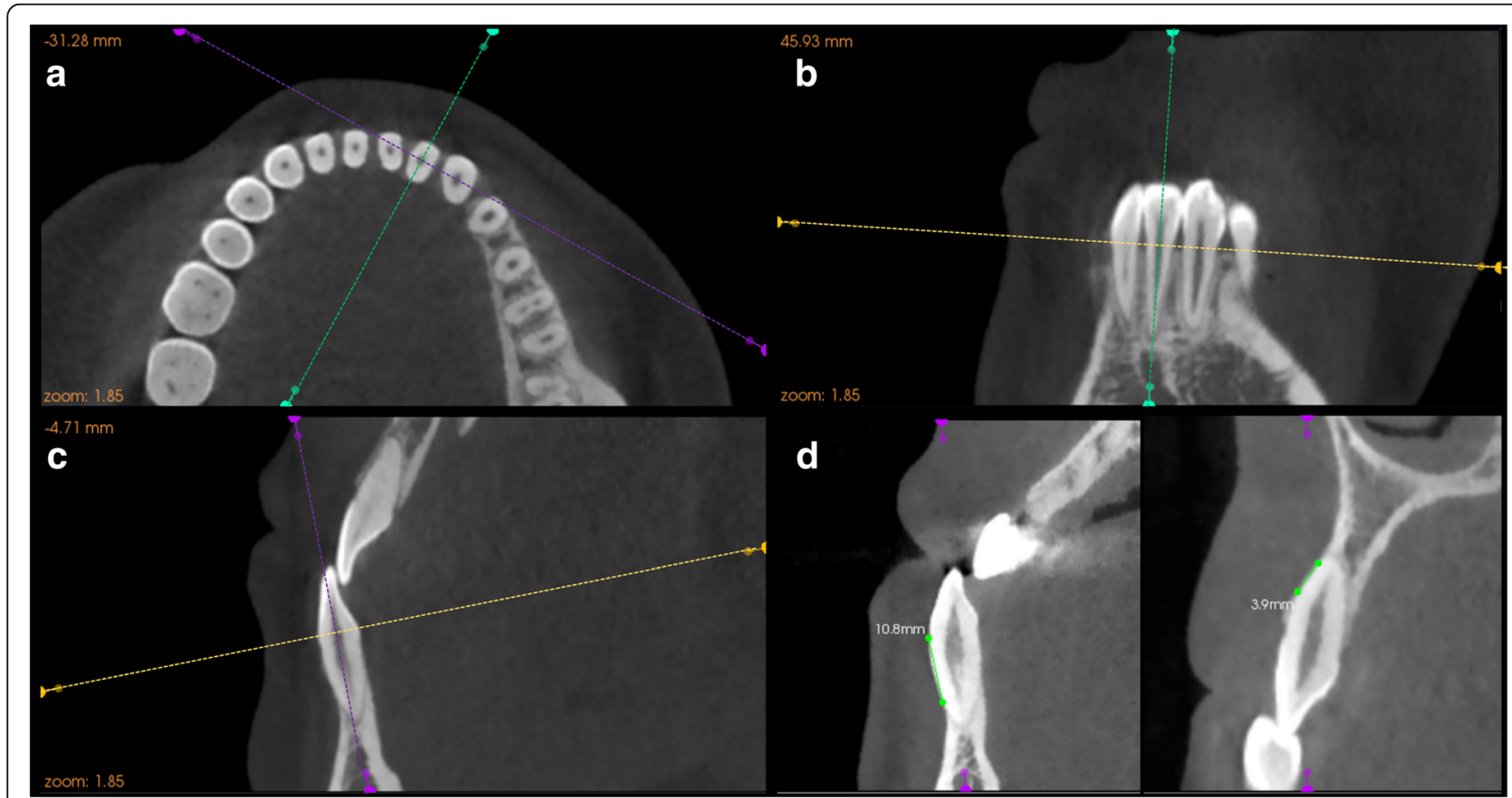

Fig. 3 Detailed procedure of locating the measurement plane of the selected tooth. The correlated planes were determined by 3 intersected guide lines with different colors representing the correlated planes, which are yellow for axial plane, green for sagittal plane, and purple for coronal plane. a Adjust the location of the axial plane by passing the yellow guideline through the CEJ of the selected tooth in both the coronal and sagittal views, then rotate the purple guideline until the intersecting line is the shortest. b Rotate the green guideline until it passes through the root apex and the midpoint of the incisal margin. $\mathbf{c}$ Rotate the purple guideline until it passes through the root apex and the cusp. $\mathbf{d}$ To ensure precise and accurate identification of anatomic structures, the largest labiolingual section displayed in the sagittal view was chosen as the measurement plane. A 10.8-mm dehiscence (left) and a 3.9-mm fenestration (right) are shown in the measurement plane 
Table 2 Definition of reference points and variables

\begin{tabular}{ll}
\hline Reference points and variables & Definition \\
\hline Dehiscence & Alveolar bone defect involving an alveolar margin $2 \mathrm{~mm}$ or greater and concurrent with a v-shaped BM \\
A & A circumscribed defect on the alveolar bone exposing the root, not involving the alveolar crest \\
B & CEJ at labial side \\
$C$ & Alveolar crest at labial side \\
D & The coronal border of a fenestration \\
$d(\mathrm{~mm})$ & The apical border of a fenestration \\
$d_{0}(\mathrm{~mm})$ & The distance between A and B \\
$d_{1}(\mathrm{~mm})$ & The distance between A and B at T0 \\
$f(\mathrm{~mm})$ & The distance between A and B at T1 \\
$f_{0}(\mathrm{~mm})$ & The distance between C and D \\
$f_{1}(\mathrm{~mm})$ & The distance between C and D at To \\
$C P D C(\mathrm{~mm})$ & The distance between C and D at T1 \\
CPFC $(\mathrm{mm})$ & $\begin{array}{l}\text { Critical point of } d \text { for dehiscence on CBCT, the value of } d \text { more than which the tooth was classified as dehiscence, } \\
\text { otherwise it was considered healthy. } \\
\text { Critical point of } f \text { for fenestration on CBCT, the value of } f \text { more than which the tooth was classified as fenestration, }\end{array}$ \\
\hline
\end{tabular}

anterior region. After the exclusion criteria-(1) craniofacial syndromes, (2) obvious pathology (cyst or tumor), (3) history of orthodontic treatment, (4) restorations on anterior teeth, (5) defective dentition or supernumerary teeth in the anterior region, or (6) periodontal disease in the anterior region-were applied, the final sample of 17 patients with 204 anterior teeth was selected.

After being fully informed of the advantages and risks of augmented corticotomy-assisted presurgical orthodontics, seven patients (having 84 teeth) who chose conventional procedures and seven patients (42 upper teeth) who accepted augmented corticotomy in the lower anterior region were allocated to control groups G1 (78 teeth) and G3 (42 teeth), representing upper and lower teeth, respectively. The seven (84 teeth) who chose our decompensation procedures were allocated to treatment groups G2 (24 teeth) and G4 (60 teeth), representing the upper and lower teeth, respectively. Screening procedures of the subjects are shown in Fig. 1. Demographic and pretreatment characteristics of the sample are shown in Table 1 . This study was approved by the independent ethics committee of Shanghai Ninth People's Hospital affiliated with Shanghai Jiao Tong University, School of Medicine (IRB NO. 201592). Informed written consent was obtained from each patient and a parent or guardian.

\section{Treatment procedures}

Periodontal therapy was conducted for all subjects 2 weeks before the brackets, and wires were engaged. Brackets and tubes were fully bonded to the second molars. The arch-wire sequence involved 0.016-in., 0.018-in., $0.018 \times$ 0.025 -in., and $0.019 \times 0.025$-in. nickel-titanium wires followed by a $0.019 \times 0.025$-in. stainless steel wire before presurgical orthodontics had been completed [16, 17, 19]. For G1 and G3 dental arches, presurgical orthodontics was carried out at 1-month intervals. For the G2 and G4 dental arches, a 0.016-in. nickel-titanium wire was engaged 1 day before augmented corticotomy, and orthodontic forces were activated at 2-week intervals. Detailed procedures of the augmented corticotomy are illustrated in Fig. 2.

\section{Image acquisition, processing, and measurement parameters}

Cone-beam computed tomography images (VG, New Tom, Verona, Italy) were obtained both before presurgical orthodontics and after postsurgical orthodontics (defined as T0 and T1, respectively). The scanning parameters for imaging were $110 \mathrm{kV}, 0-20 \mathrm{~mA}$ (achieved automatically by setting the grey values at 16 bits), exposure time of $5.4 \mathrm{~s}$, and a 12-in. field of view (F-mode). These settings produced a voxel size of $0.125 \mathrm{~mm}$. The settings were the same as those used for the orthodontic diagnosis and treatment planning in the Department of Oral \& Cranio-Maxillofacial Science, Ninth People's Hospital. The acquired CBCT data were imported into the integrated image processing software (Kodak Dental Imaging Software 3D Module V2.4, Eastman Kodak, Rochester, NY, USA). Anterior teeth that fitted our criteria were chosen as study samples, and the largest labiolingual section was defined as the measurement plane. Figure 3 illustrates the detailed protocol of locating the measurement plane. The description of landmarks and measurement variables are modifications of those obtained in the report by Sun et al. [15] (Table 2 and Fig. 4). 


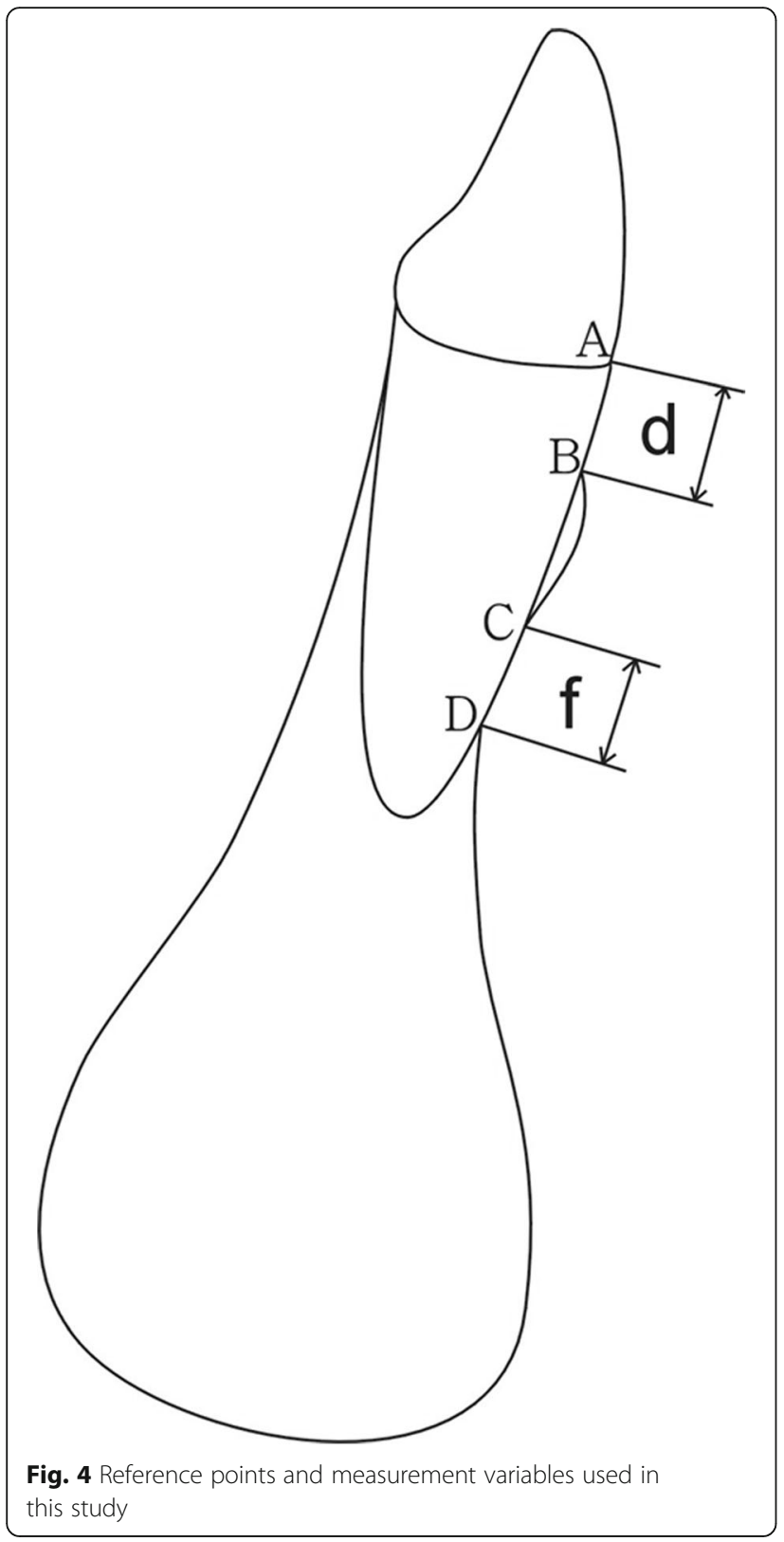

According to Sun et al.'s study [15], we set the critical point for dehiscence on the CBCT (CPDC) at $2 \mathrm{~mm}$ and the critical point for fenestration on the CBCT (CPFC) at $2.2 \mathrm{~mm}$, which means that when $d$ was more than 2 $\mathrm{mm}$ on the CBCT, the defect was classified as dehiscence; when $f$ was more than $2.2 \mathrm{~mm}$ on the CBCT, it was classified as fenestration. To limit experimental bias, dehiscences and fenestrations were re-examined on the CBCT image, the $d$ and $f$ of all teeth at T0 and T1 were re-measured in 4 weeks, and the resulting mean values were used. All measurements were made by the same operator (L.S.).

Classification of the transition for dehiscence and fenestration after treatment is shown in Table 3.
Table 3 The classification of the transition for dehiscence and fenestration after treatment

\begin{tabular}{lll}
\hline \multicolumn{1}{c}{ Transition degree } & & Definition \\
\hline$d_{0} \leq 2 \mathrm{~mm}$ & 1 & $d_{1} \leq 2 \mathrm{~mm}$ (maintain) \\
& 2 & $d_{1}>2 \mathrm{~mm}$ (worsen) \\
$d_{0}>2 \mathrm{~mm}$ & 1 & $d_{1} \leq 2 \mathrm{~mm}$ (cure) \\
& 2 & $2<d_{1}<d_{0}$ (improve) \\
& 3 & $d_{1}=d_{0}$ (maintain) \\
& 4 & $d_{1}>d_{0}$ (worsen) \\
$f_{0} \leq 2.2 \mathrm{~mm}$ & 1 & $f_{1} \leq 2.2 \mathrm{~mm}$ (maintain) \\
& 2 & $f_{1}>2.2 \mathrm{~mm}$ (worsen) \\
$f_{0}>2.2 \mathrm{~mm}$ & 1 & $f_{1} \leq 2.2 \mathrm{~mm}$ (cure) \\
& 2 & $2.2<f_{1}<f_{0}$ (improve) \\
& 3 & $f_{1}=f_{0}$ (maintain) \\
& 4 & $f_{1}>f_{0}$ (worsen) \\
\hline
\end{tabular}

\section{Statistical analysis}

All statistical analyses were performed with the Statistical Package for the Social Sciences (version 16.0; SPSS, Chicago, IL). $d_{0}$ and $d_{1}$ of all groups were compared by Student's $t$ tests, so were $f_{0}$ and $f_{1}$ of all groups. Mixed model $t$ tests were performed to compare $d_{1}-d_{0}$ and $f_{1}$ $-f_{0}$ of both control and treatment groups. Chi-square test and Wilcoxon rank sum test were performed to compare the transition degree of both control and treatment groups. Intra-operator reliability was assessed by calculating the intra-class correlation coefficient (ICC) between measurements collected at both times. The significance level was set at a 2 -tailed $P$ value of 0.05 .

\section{Results}

Table 4 shows the ICC values for $d$ and $f$ before and after treatment (four continuous variables: $d_{0}, d_{1}, f_{0}$, and $f_{1}$ ) indicating that our measurements had excellent reliability.

Table 5 shows the changes of $d$ and $f$ after treatment. For G1, there was a significant increase in $d(P<0.01)$ and an insignificant increase in $f$. For G2, there was an insignificant decrease in both $d$ and $f$. For G3, there was a significant increase in $d(P<0.01)$ and a significant decrease in $f(P<0.01)$. For $\mathrm{G} 4$, there was a significant decrease in both $d$ and $f(P<0.01)$.

Table 6 shows the comparison of changes of $d\left(d_{1}-\right.$ $\left.d_{0}\right)$ and $f\left(f_{1}-f_{0}\right)$ between the control and treatment groups. For the upper teeth, $d_{1}-d_{0}$ was statistically different between both groups $(P<0.01)$ while $f_{1}-f_{0}$ was not statistically different. For the lower teeth, $d_{1}-d_{0}$ was statistically different between both groups $(P<0.01)$ while $f_{1}-f_{0}$ was not statistically different.

Table 7 compares the degree of transition for dehiscence and fenestration between the control and treatment groups. For teeth having no dehiscence before 
Table 4 Intraoperator reliability for $d$ and $f$ measurements before treatment (T0) and after treatment (T1) by means, standard deviations, and ICC

\begin{tabular}{|c|c|c|c|c|c|c|}
\hline \multirow{2}{*}{ 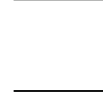 } & \multicolumn{3}{|l|}{$\mathrm{T} 1$} & \multicolumn{3}{|l|}{$\mathrm{T} 2$} \\
\hline & Measurement 1 & Measurement 2 & & Measurement 1 & Measurement 2 & \\
\hline$n=204$ & Mean \pm SD $(\mathrm{mm})$ & Mean \pm SD $(\mathrm{mm})$ & ICC & Mean \pm SD $(\mathrm{mm})$ & Mean \pm SD $(\mathrm{mm})$ & ICC \\
\hline$d$ & $3.51 \pm 3.02$ & $3.55 \pm 3.08$ & 0.995 & $3.77 \pm 3.16$ & $3.71 \pm 3.10$ & 0.992 \\
\hline$f$ & $1.56 \pm 1.79$ & $1.60 \pm 1.85$ & 0.994 & $1.07 \pm 1.57$ & $1.05 \pm 1.53$ & 0.997 \\
\hline
\end{tabular}

treatment, G2 and G4 had a better transition than did G1 or G3 $(P<0.05)$. For those having dehiscence before treatment, G4 had a better transition than did G3 $(P<$ 0.01 ), but there was no statistically significant difference in the transition between G1 and G2. For teeth having no fenestration before treatment, there was no statistically significant difference in the transition between the control and treatment groups. For those having fenestration before treatment, G4 had a better transition than did G3 $(P<0.01)$, but there was no statistically significant difference between G1 and G2.

\section{Discussion}

The detection of alveolar bone dehiscence and fenestration in vivo can be achieved in three possible ways: inspecting during mucogingival surgery, exploring with the periodontal probe, and radiograph. Since patients undergoing periodontal surgery are very few and carefully selected, the first method cannot be widely applied. Using a periodontal probe and taking traditional X-rays are unreliable and unsatisfactory. Timock et al. [13] found strong agreement between $\mathrm{CBCT}$ and direct measurements for buccal bone height and thickness, which speaks for the accuracy and reliability of CBCT in measuring those parameters. Researches have shown that computed tomography and $\mathrm{CBCT}$ are highly effective in detecting artificially created bony defects and naturally occurring alveolar dehiscences and fenestrations on skulls [14, 22-25]. Leung et al. [14] reported that under a $0.38-\mathrm{mm}$ voxel size, the diagnostic value of CBCT for detecting buccal defects was high for fenestrations: both sensitivity and specificity were about 0.80 ; for dehiscences, the specificity was high at 0.95 , but the sensitivity was low at 0.40 . Our previous study [15] showed that under a $0.125-\mathrm{mm}$ voxel size, while using the best critical points $(\mathrm{CPDC}=2.2 \mathrm{~mm}, \mathrm{CPFC}=2.2 \mathrm{~mm}$ ), both sensitivity and specificity for dehiscence and fenestration were acceptable (both about 0.8 ). The PV+ value for detecting dehiscence was 0.84 , and the PV- value for detecting dehiscence and fenestration was 0.79 and 0.98 , respectively, which means that $84 \%$ of the detected dehiscences truly existed and most of the healthy teeth diagnosed by CBCT were truly healthy. On the other hand, the PV+ value for detecting fenestration was 0.21 , meaning that when a fenestration was detected, it was a true fenestration about $20 \%$ of the time. By using our CBCT settings and methods, although there was a systematic overestimation of the CBCT measurements, its diagnostic value in detecting alveolar bone dehiscences and fenestrations was still acceptable. It can be concluded that at present the only satisfactory method of detecting alveolar bone dehiscence and fenestration in vivo before treatment is the CBCT method.

This study showed a higher reliability of measuring the vertical diameter of dehiscences and fenestrations by CBCT, with ICC values of 0.992 and 0.997 . The time interval between the first and second measurements was 4 weeks. Our results were similar to that reported by Leung et al. [14] (0.891-0.994) and Sun et al. [15] (0.994-0.0996).

For class III malocclusion, presurgical orthodontic decompensation requires uprighting the proclined maxillary incisors and retroclining the mandibular incisors to more normal axial inclinations. Large amounts of dental decompensation might be associated with a higher tendency to develop gingival recessions [26-28]. However, there are only a few studies on alveolar bone change after orthodontic treatment. Wehrbein et al. [29] evaluated the alveolar bone of a deceased patient who had undergone orthodontic treatment and found severe bone loss on the labial and lingual cortical plates. Lee et al. [9] evaluated the alveolar bone loss around lower incisors

Table 5 Changes of $d$ and $f$ after treatment

\begin{tabular}{|c|c|c|c|c|c|c|c|c|}
\hline & \multicolumn{2}{|l|}{ G1 } & \multicolumn{2}{|l|}{ G2 } & \multicolumn{2}{|l|}{ G3 } & \multicolumn{2}{|l|}{ G4 } \\
\hline & Mean \pm SD & $P$ & Mean \pm SD & $P$ & Mean \pm SD & $P$ & Mean \pm SD & $P$ \\
\hline$d_{0}$ & $1.873 \pm 1.841$ & 0.009 & $3.346 \pm 2.198$ & 0.062 & $5.283 \pm 3.199$ & 0.005 & $4.520 \pm 3.440$ & 0.009 \\
\hline$d_{1}$ & $2.763 \pm 3.414$ & & $2.546 \pm 1.214$ & & $6.826 \pm 2.691$ & & $3.320 \pm 2.015$ & \\
\hline$f_{0}$ & $1.377 \pm 1.610$ & 0.240 & $1.008 \pm 1.398$ & 0.350 & $1.674 \pm 1.795$ & 0.002 & $2.015 \pm 2.143$ & 0.000 \\
\hline$f_{1}$ & $1.659 \pm 2.227$ & & $0.738 \pm 0.834$ & & $0.888 \pm 1.540$ & & $0.527 \pm 0.207$ & \\
\hline
\end{tabular}


Table 6 Comparison of $d_{1}-d_{0}$ and $f_{1}-f_{0}$ for samples between control and treatment groups

\begin{tabular}{|c|c|c|c|c|c|c|c|c|c|c|}
\hline \multicolumn{3}{|c|}{$\mathrm{G1}$} & \multicolumn{2}{|l|}{$\mathrm{G} 2$} & \multirow[b]{2}{*}{ P } & \multicolumn{2}{|l|}{ G3 } & \multicolumn{2}{|l|}{ G4 } & \multirow[b]{2}{*}{ P } \\
\hline & Mean & SD & Mean & SD & & Mean & SD & Mean & SD & \\
\hline$d_{1}-d_{0}$ & 0.89 & 2.928 & -0.8 & 1.551 & 0.002 & 1.543 & 3.339 & -1.2 & 3.433 & 0.000 \\
\hline$f_{1}-f_{0}$ & 0.282 & 2.105 & -0.271 & 1.390 & 0.141 & -0.786 & 1.54 & -1.488 & 2.172 & 0.059 \\
\hline
\end{tabular}

incurred during surgical orthodontic treatment in 25 individuals with mandibular prognathism. They concluded that excessive forward movement of lower incisors during presurgical orthodontic treatment could cause alveolar bone loss. Our results showed the same tendency: After traditional treatment, dehiscence of both upper and lower anterior teeth worsened, while fenestration of lower anterior teeth improved. For lower anterior teeth, because root lingual torque control might mitigate the prominence of the apexes, fenestration improved.

The aim of our study was to evaluate on CBCT the changes of alveolar dehiscence and fenestration after augmented corticotomy-assisted orthodontic treatment compared with traditional pre-surgical orthodontics, both quantitatively and qualitatively. Our results suggest that, after augmented corticotomy-assisted orthodontic treatment, both dehiscence and fenestration improved for the lower anterior teeth. Compared with traditional treatment, the augmented corticotomy-assisted orthodontic treatment showed more healing effect for dehiscences than for fenestration in both upper and lower anterior teeth. Table 7 indicates that, compared with traditional treatment, augmented corticotomy-assisted orthodontic treatment has the potential to protect both upper and lower healthy anterior teeth against dehiscence. The results also suggest that augmented corticotomy-assisted orthodontic treatment had a healing effect on lower anterior teeth region both for dehiscence and fenestration.

Table 7 Comparison of transition degree for dehiscence and fenestration between control and treatment groups

\begin{tabular}{|c|c|c|c|c|c|c|c|c|}
\hline \multicolumn{3}{|c|}{ Transition degree } & \multirow{2}{*}{$\frac{\mathrm{G} 1}{41}$} & \multirow{2}{*}{$\frac{G 2}{2}$} & \multirow{2}{*}{$\begin{array}{l}P \\
0.0412\end{array}$} & \multirow{2}{*}{$\frac{\mathrm{G} 3}{1}$} & \multirow{2}{*}{$\frac{G 4}{10}$} & \multirow{2}{*}{$\frac{P}{0.0205}$} \\
\hline Dehiscence & $d_{0} \leq 2$ & 1 & & & & & & \\
\hline & & 2 & 10 & 3 & & 7 & 6 & \\
\hline & $d_{0}>2$ & 1 & 6 & 7 & 0.1193 & 2 & 7 & 0.0039 \\
\hline & & 2 & 3 & 7 & & 7 & 20 & \\
\hline & & 3 & 1 & 1 & & 2 & 1 & \\
\hline & & 4 & 11 & 4 & & 23 & 16 & \\
\hline \multirow[t]{6}{*}{ Fenestration } & $f_{0} \leq 2.2$ & 1 & 41 & 19 & 0.126 & 24 & 36 & 0.080 \\
\hline & & 2 & 10 & 1 & & 1 & 1 & \\
\hline & $f_{0}>2.2$ & 1 & 6 & 3 & 0.208 & 11 & 23 & 0.002 \\
\hline & & 2 & 7 & 0 & & 4 & 0 & \\
\hline & & 3 & 0 & 0 & & 0 & 0 & \\
\hline & & 4 & 8 & 1 & & 2 & 0 & \\
\hline
\end{tabular}

Just as the most probable etiologic cause of alveolar dehiscence and fenestration is a combination of prominent roots and a thin alveolar bone plate, the most probable mechanism for healing is the combination of root control and the augmented bone graft. As previously mentioned, recent studies have reported that an increase in alveolar bone thickness which covers the root surface might be explained by the same mechanism. That is, first, either the bone graft material augmented the hard tissue overlying the root surface or it eliminated instantly the pre-existing defect. Then, the increased alveolar volume and a more structurally complete periodontium allowed us to move the teeth safely. After decompensation and torque control during presurgical orthodontics, the position and inclination of the teeth were controlled and the roots were set in the center of the alveolar process.

However, one important question remains: Can true periodontal regeneration be achieved on the root surface? Is the bone augmentation seen on $\mathrm{CBCT}$ real bone tissue, or just bone material, or a mixture of both? Unlike Wilcko's [16] study, we did not perform the re-entrance to obtain the biopsy, and the duration was relatively short, especially for the treatment groups. Experimental animal studies and long-term follow-up are required in the future. Our study also had other limitations. Our subjects were all skeletal class III malocclusions, and we collected only anterior teeth, because alveolar dehiscences and fenestrations are most common in the anterior region of class III malocclusions. In addition, our sample size was not large enough. More subjects having different kinds of malocclusions should be accumulated, and both anterior and posterior teeth should be included in the future.

\section{Conclusions}

For skeletal class III patients, augmented corticotomyassisted orthodontic treatment is a promising method to improve the alveolar bone dehiscence and fenestration of lower anterior teeth, and it also has the potential to protect both lower and upper anterior teeth from dehiscence. 


\section{Acknowledgements}

This work was supported by the Science Foundation of Shanghai Municipal Commission of Health and Family Planning (Grant No. 20164Y0130).

\section{Funding}

This work was supported by the Science Foundation of Shanghai Municipal Commission of Health and Family Planning (Grant No. 20164Y0130).

\section{Availability of data and materials}

Data is included in the form of tables in the study. Please contact author for data requests.

\section{Authors' contributions}

$\mathrm{BF}$ and GS were responsible for the study design. BF was responsible for the administration, critical revision for important intellectual content, and final approval of the article. LS, BW, and LY contributed to the literature search and manuscript review. LS and BW contributed to the drafting of the manuscript. LS selected the patients and contributed to the acquisition of data. LZ performed the statistical analysis and data analysis. All authors read and approved the final manuscript.

\section{Ethics approval and consent to participate}

All the participants' guardian informed consents were obtained. The present study was approved by the independent ethics committee of Shanghai Ninth People's Hospital affiliated with Shanghai Jiao Tong University, School of Medicine (IRB number: 201592).

\section{Consent for publication}

Not applicable.

\section{Competing interests}

The authors declare that they have no competing interests.

\section{Publisher's Note}

Springer Nature remains neutral with regard to jurisdictional claims in published maps and institutional affiliations.

\section{Author details}

'Department of Orthodontics, Shanghai Stomatological Hospital, 1258 Fuxing Rd, 2nd Floor, Shanghai 200000, China. '2Department of Orthodontics, Ninth People's Hospital, College of Stomatology, Shanghai Jiao Tong University School of Medicine, 500 Quxi Rd, 7th Floor, Shanghai 200000, China. ${ }^{3}$ Department of Oral \& Cranio -Maxillofacial Science, Ninth People's Hospital, College of Stomatology, Shanghai Jiao Tong University School of Medicine, 500 Quxi Rd, 4th Floor, Shanghai 200000, China. ${ }^{4}$ Department of Biostatistics, College of Stomatology, Shanghai Jiao Tong University School of Medicine, 227 South Chongqing Rd, Shanghai 200000, China.

\section{Received: 29 October 2018 Accepted: 7 January 2019}

\section{Published online: 18 February 2019}

\section{References}

1. Yagci A, Veli I, Uysal T, Ucar Fl, Ozer T, Enhos S. Dehiscence and fenestration in skeletal class I, II, and III malocclusions assessed with cone-beam computed tomography. Angle Orthod. 2012;82:67-74.

2. Nahm KY, Kang JH, Moon SC, Choi YS, Kook YA, Kim SH, et al. Alveolar bone loss around incisors in class I bidentoalveolar protrusion patients: a retrospective three-dimensional cone beam CT study. Dentomaxillofac Radiol. 2012:41:481-8.

3. Kim Y, Park JU, Kook YA. Alveolar bone loss around incisors in surgical skeletal class III patients. Angle Orthod. 2009;79:676-82.

4. Evangelista K, Vasconcelos Kde F, Bumann A, Hirsch E, Nitka M, Silva MA. Dehiscence and fenestration in patients with class I and class II division 1 malocclusion assessed with cone-beam computed tomography. Am J Orthod Dentofac Orthop. 2010;138(2):133 e131-7; discussion 133-5.

5. Sun $L Y$, Wang $B$, Fang $B$. The prevalence of dehiscence and fenestration on anterior region of skeletal class III malocclusions:a cone-beam CT study. Shanghai Kou Qiang Yi Xue. 2013;22:418-22.

6. Lost C. Depth of alveolar bone dehiscences in relation to gingival recessions. J Clin Periodontol. 1984;11:583-9.
7. Artun J, Urbye KS. The effect of orthodontic treatment on periodontal bone support in patients with advanced loss of marginal periodontium. Am J Orthod Dentofac Orthop. 1988;93:143-8.

8. Wennstrom JL, Stokland BL, Nyman S, Thilander B. Periodontal tissue response to orthodontic movement of teeth with infrabony pockets. Am J Orthod Dentofac Orthop. 1993;103:313-9.

9. Lee KM, Kim YI, Park SB, Son WS. Alveolar bone loss around lower incisors during surgical orthodontic treatment in mandibular prognathism. Angle Orthod. 2012;82:637-44.

10. Artun J, Krogstad O. Periodontal status of mandibular incisors following excessive proclination. A study in adults with surgically treated mandibular prognathism. Am J Orthod Dentofac Orthop. 1987;91:225-32.

11. Wang B, Shen GF, Fang B, Sun LY, Wu Y, Jiang LY, et al. Exploration for micro-osteotomy assisted orthodontic treatment of skeletal class III malocclusions with alveolar hypoplasia in the lower anterior region. Shanghai Kou Qiang Yi Xue. 2012;21:535-40.

12. Coscia G, Coscia V, Peluso V, Addabbo F. Augmented corticotomy combined with accelerated orthodontic forces in class III orthognathic patients: morphologic aspects of the mandibular anterior ridge with cone-beam computed tomography. J Oral Maxillofac Surg. 2013;71(10):1760 e1761-9.

13. Timock A, Cook VC, McDonald T, Leo MC, Crowe J. Accuracy and reliability of buccal bone height and thickness measurements from cone-beam computed tomography imaging. Am J Orthod Dentofac Orthop. 2011; 140(5):734-44.

14. Leung CC, Palomo L, Griffith R, Hans MG. Accuracy and reliability of conebeam computed tomography for measuring alveolar bone height and detecting bony dehiscences and fenestrations. Am J Orthod Dentofac Orthop. 2010;137:S109-19.

15. Sun L, Zhang L, Shen G, Wang B, Fang B. Accuracy of cone-beam computed tomography in detecting alveolar bone dehiscences and fenestrations. Am J Orthod Dentofac Orthop. 2015;147:313-23.

16. Wilcko MT, Wilcko WM, Pulver JJ, Bissada NF, Bouquot JE. Accelerated osteogenic orthodontics technique: a 1-stage surgically facilitated rapid orthodontic technique with alveolar augmentation. J Oral Maxillofac Surg. 2009:67:2149-59.

17. Amit G, Jps K, Pankaj B, Suchinder S, Parul B. Periodontally accelerated osteogenic orthodontics (PAOO) - a review. J Clin Exp Dent. 2012:4:e292-6.

18. Gantes B, Rathbun E, Anholm M. Effects on the periodontium following corticotomy-facilitated orthodontics. Case reports. J Periodontol. 1990;61:234-8.

19. Wang B, Shen G, Fang B, Yu H, Wu Y, Sun L. Augmented corticotomyassisted surgical orthodontics decompensates lower incisors in class III malocclusion patients. J Oral Maxillofac Surg. 2014;72:596-602.

20. Ma ZG, Yang C, Xi QY, Ye ZX, Zhang SY, Abdelrehem A. A novel surgical technique for augmented corticotomy-assisted orthodontics: bone grafting with periosteum. J Oral Maxillofac Surg. 2016;74:170-80.

21. Yu H, Jiao F, Wang B, Shen SG. Piezoelectric decortication applied in periodontally accelerated osteogenic orthodontics. J Craniofac Surg. 2013; 24:1750-2.

22. Misch KA, Yi ES, Sarment DP. Accuracy of cone beam computed tomography for periodontal defect measurements. J Periodontol. 2006;77:1261-6.

23. Pinsky HM, Dyda S, Pinsky RW, Misch KA, Sarment DP. Accuracy of threedimensional measurements using cone-beam CT. Dentomaxillofac Radiol. 2006;35:410-6

24. Mengel R, Candir M, Shiratori K, Flores-de-Jacoby L. Digital volume tomography in the diagnosis of periodontal defects: an in vitro study on native pig and human mandibles. J Periodontol. 2005;76:665-73.

25. Fuhrmann R. Three-dimensional interpretation of alveolar bone dehiscences. An anatomical-radiological study--part I. J Orofac Orthop. 1996;57:62-74.

26. Joss-Vassalli I, Grebenstein C, Topouzelis N, Sculean A, Katsaros C. Orthodontic therapy and gingival recession: a systematic review. Orthod Craniofac Res. 2010:13:127-41.

27. Djeu G, Hayes C, Zawaideh S. Correlation between mandibular central incisor proclination and gingival recession during fixed appliance therapy. Angle Orthod. 2002;72:238-45.

28. Yared KF, Zenobio EG, Pacheco W. Periodontal status of mandibular central incisors after orthodontic proclination in adults. Am J Orthod Dentofac Orthop. 2006;130(1):6 e1-8.

29. Wehrbein H, Bauer W, Diedrich P. Mandibular incisors, alveolar bone, and symphysis after orthodontic treatment. A retrospective study. Am J Orthod Dentofac Orthop. 1996;110:239-46. 